\title{
Management of Isolated Yew Berry Toxicity With Sodium Bicarbonate: A Case Report in Treatment Efficacy
}

\author{
Jessica E. Pierog, DO ${ }^{a}$, Bryan Kane, MD ${ }^{a}$, Kathleen Kane, $\mathrm{MD}^{a}$, J. Ward Donovan, $\mathrm{MD}^{b}$ \\ aLehigh Valley Health Network, Emergency Medicine Residency, Allentown \& Bethlehem, PA \\ bPinnacleHealth Toxicology Center, Harrisburg Hospital, Harrisburg, PA
}

\begin{abstract}
Introduction: Taxus species are known to be toxic and may result in significant dysrhythmias. Treatment of taxus induced cardiac dysrhythmias is based largely on case reports. We describe a case of a 24-year-old male with Taxus cuspidate (yew berry) toxicity initially treated with amiodarone bolus and infusion and subsequently managed with sodium bicarbonate boluses and continuous infusion.

Case Report: The patient was found at home by his parents with witnessed "seizure-like" activity 2 hours after reportedly chewing and swallowing 168 yew seeds. The initial prehospital rhythm strip demonstrated ventricular tachycardia (VT); the patient was hypotensive with fluctuating levels of alertness. Prehospital cardioversion was attempted without success.

Staff at the local presenting emergency department (ED) consulted toxicology for management of the presumed yew berry ingestion, complicated by cardiac dysrhythmias and mental status change with seizure. Amiodarone 300-mg IV and diazepam 5-mg IV were given. Cardioversion was attempted 4 times without change in the wide complex tachycardia, presumed to be VT, at a rate of 166 . An amiodarone drip at $1 \mathrm{mg} / \mathrm{min}$ was initiated. The patient was transferred to an intensive care unit (ICU) at a regional toxicology center.

On arrival to the toxicology center the patient was alert and verbally appropriate without complaints. Initial heart rate was 76 and regular with premature ventricular contractions (PVCs). A wide complex tachycardia associated with hypotension recurred; however, normal mental status was maintained. A bolus of $100 \mathrm{mEq}$ of sodium bicarbonate (NaHCO3) was given intravenously followed by sodium bicarbonate infusion at $37.5 \mathrm{mEq} / \mathrm{hr}$. The amiodarone drip was discontinued. Subsequent electrocardiograms (EKG's) revealed a prolonged, but steadily narrowing QRS complex. Ultimately, the QRS complex closed to $92 \mathrm{~ms}$, with a rate of 94 , PR 154 and a QT/QTc of 390/487.

Conclusion: This case describes successful treatment of an isolated Taxus cuspidate (yew berry) ingestion with significant toxicity initially with amiodarone bolus and infusion. Due to lack of significant change in telemetry recordings with amiodarone, treatment with sodium bicarbonate bolus and infusion was initiated. While the QRS narrowed significantly temporally related to the bicarbonate, it is difficult to determine if correction of the cardiac dysrhythmias was solely due to the sodium bicarbonate, or the synergism of sodium bicarbonate and amiodarone, or possibly spontaneous improvement due to taxine clearance. One should use caution while drawing conclusions from a single case; however, based on the clinical improvement of this patient, both with EKG recordings and vital signs, this report would suggest that isolated Taxus cuspidate ingestion from yew berry plants can be treated with sodium bicarbonate.
\end{abstract}

Keywords: taxus toxicity, yew berry toxicity, digoxin-specific antibody fragments, sodium bicarbonate Notes: The information contained in the case report has not been presented previously.

There was no outside funding of any kind used for this study.

Corresponding Author: Jessica E. Pierog, DOa, Lehigh Valley Hospital, Allentown \& Bethlehem PA. Email: jessicapierog@hotmail.com 


\section{INTRODUCTION}

Taxus species are known to be toxic. Taxus ingestion can result in severe dysrhythmias and mortality from resulting abnormalities in cardiac conduction and failure to detect clinical features in time. Treatment of taxus-induced cardiac dysrhythmias is based largely on case reports. Lidocaine has been the treatment of choice for dysrhythmias associated with taxus toxicity, as it is a recognized treatment for ventricular dysrhythmias not associated with poisoning [1]. We describe the case of a 24-year-old male with Taxus cuspidate (yew berry) toxicity manifesting as unstable ventricular tachycardia associated with intermittent mental status changes. He was initially treated with amiodarone and subsequently managed with a sodium bicarbonate continuous infusion.

\section{CASE REPORT}

A 24-year-old male was found at home by his parents, who witnessed "seizure-like" activity. They immediately called for emergency medical services (EMS). EMS personnel found the patient seated, alert, and diaphoretic. The initial prehospital rhythm strip demonstrated ventricular tachycardia with a rate of 186 , QRS 172 msec, QT/QTc 270/475 msec. Prehospital cardioversion was attempted, as the patient was also hypotensive; this attempt was unsuccessful. On arrival to the local emergency department (ED), the patient complained of chest pain, palpitations, nausea, vomiting, and abdominal pain. The patient alleged to have purposely ingested, solely, 168 yew seeds 2 hours prior to arrival at the ED in a suicide attempt. The patient also claims to have ingested ipecac with resultant vomiting. His past medical history included depression, insomnia, schizoaffective disorder, and a previous suicide attempt with poison hemlock. Illicit drug or alcohol use was denied. Outpatient medications included zaleplon, gabapentin, and clozapine. Vital signs at admission to the local ED were: BP 59/31, pulse oximetry $87 \%$ on room air, temperature $97.1 \mathrm{~F}$, pulse $170-200$, and RR 20. Electrocardiogram (EKG) at this time is shown in Figure 1. The patient was in no acute distress, but was diaphoretic. Cardiovascular, pulmonary, and abdominal examinations were unremarkable, making it unclear if his hypoxia was related to poor peripheral perfusion or respiratory compromise from another source. Intermittent 15 -second, generalized tonic clonic seizure activity occurred, with occasional unresponsive episodes lasting several minutes. Staff in the local ED was uncertain if these were seizures followed by postictal episodes. Pupils were $3 \mathrm{~mm}$ bilaterally and would dilate to $6 \mathrm{~mm}$ during the unresponsive spells.

The toxicology service was consulted for management of the presumed yew berry ingestion, complicated by cardiac dysrhythmias. On the recommendation of the toxicology center, the local ED administered amiodarone 300-mg intravenous (IV) bolus to treat the dysrhythmia and diazepam 5-mg IV bolus to treat the presumed seizure activity. Decontamination with oral charcoal was not recommended given the time course and the patient's altered mental status. Telemetry was recorded and is shown in Figure 2 . No clinical change was noted immediately after infusion. As the initial hospital had made no further attempts at cardioversion, after administration of antiarrhythmic medication, 4 at-

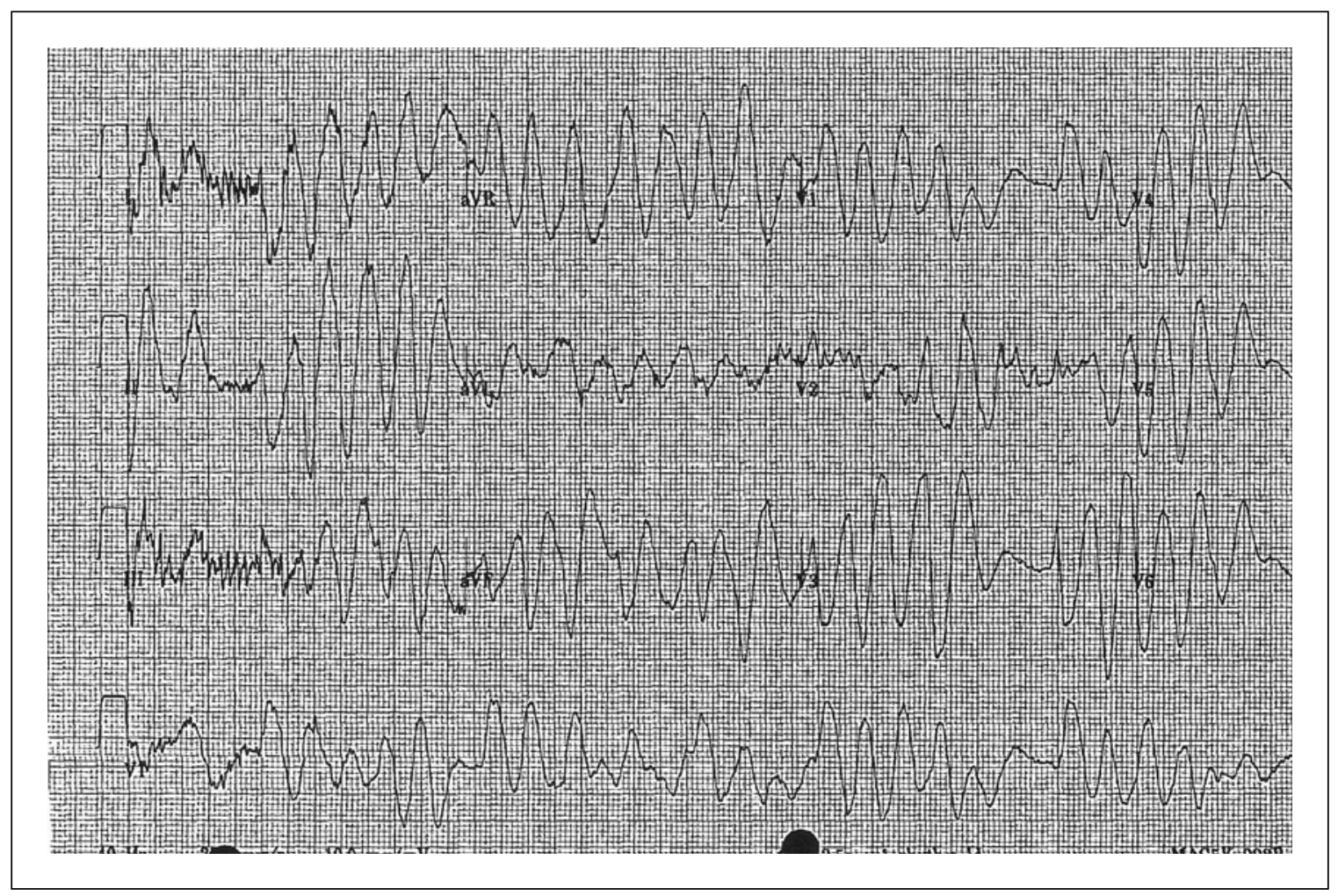

Figure 1: Initial EKG from presenting hospital 


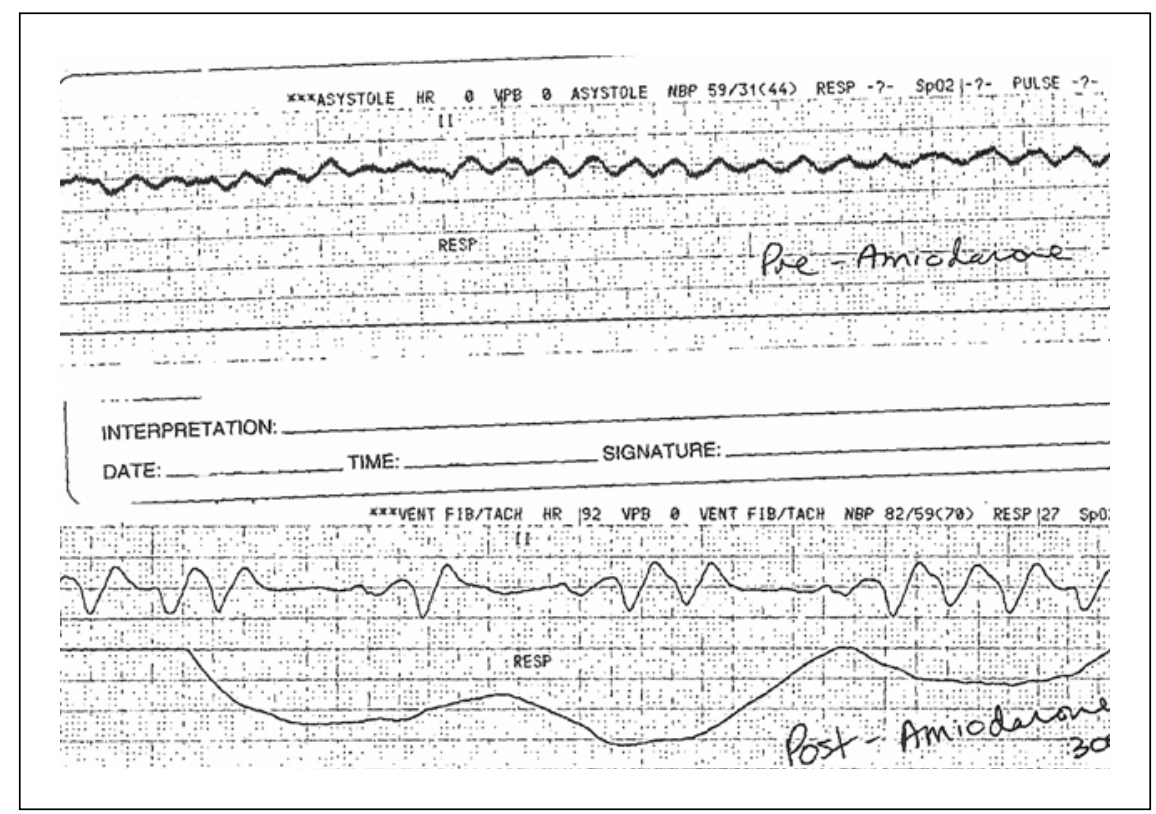

Figure 2: Telemetry from presenting hospital-before and after administration of amiodarone 300-mg IV bolus

tempts were made to cardiovert the patient. A wide complex tachycardia persisted at a rate of 166 , with a corresponding blood pressure of 77/32. An amiodarone continuous infusion (1 mg/ minute) was initiated. The patient was transported via helicopter to a regional toxicology center approximately 3 hours after arrival in the local ED.

On arrival to the intensive care unit (ICU) at the regional toxicology center, the patient was alert and coherent, without com- plaints. No further generalized motor activity or unresponsive episodes had been noted in transport. Initial vital signs were: $\mathrm{BP}$ 104/54, pulse 76 and regular, RR 14, and temperature $36.1 \mathrm{C}$. Chest $\mathrm{x}$-ray was normal. The patient was continued on maintenance IV fluids ( $0.9 \%$ normal saline at $150 \mathrm{ml} /$ hour) and amiodarone infusion ( $1 \mathrm{mg} / \mathrm{min})$. The initial toxicology center EKG is shown in Figure 3. Six hours after arrival in the ICU, a bolus of 100 mEq of $\mathrm{NaHCO} 3$ ( 2 amps of $8.4 \%$ sodium bicarbonate) was given

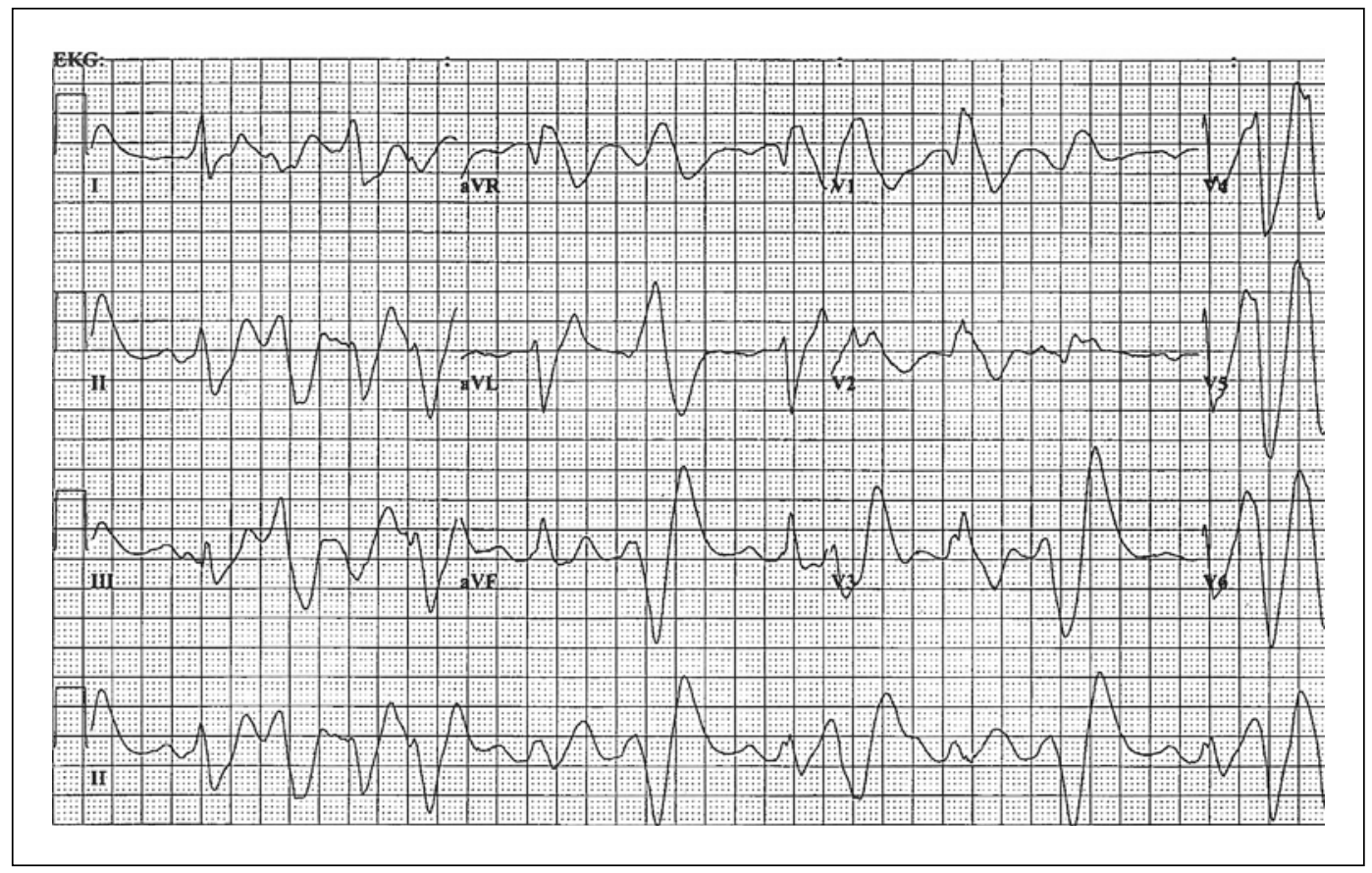

Figure 3: EKG upon arrival to toxicology ICU 
IV. Telemetry showed a narrowing QRS within minutes of the bicarbonate administration. A sodium bicarbonate infusion was initiated at $250 \mathrm{ml} /$ hour (37.5 mEq/hour). The patient was continued on maintenance IV fluids at a rate of $250 \mathrm{ml} /$ hour of normal saline. Arterial blood gas (ABG) \#1 was drawn shortly after. All ABG values are presented in Table 1. At this time, the amiodarone drip was written to be discontinued; however, because of a transcription error, the bicarbonate and amiodarone drips coexisted for approximately 4 hours. A follow-up EKG is shown in Figure 4 and correlates with ABG \#2. Of note, the patient also received metoclopramide for nausea, morphine for back pain, heparin subcutaneously for prophylaxis against deep vein thrombosis, and IV pantoprazole for gastrointestinal prophylaxis. Subsequent EKGs revealed a prolonged, but steadily narrowing QRS complex; ABG \#3 was drawn while on the infusion. The sodium bicarbonate continuous infusion was continued overnight, after which his QRS remained $<100 \mathrm{msec}$, his mental status returned to baseline, his vital signs were stable, and he no longer had ventricular dysrhythmias. An EKG at the cessation of the bicarbonate drip showed a rate of 91, QRS duration of $84 \mathrm{msec}$, and QT/QTc $380 / 467 \mathrm{msec}$; this correlates with ABG \#4. The patient's discharge EKG 56 hours after admission to the toxicology center is shown in Figure 5. He was discharged to an inpatient psychiatric facility; he was ambulating without assistance, able to perform all activities of daily living, intact neurologically, and hemodynamically stable.

On presentation to the toxicology center, the patient had acute renal insufficiency with a creatinine of $1.6 \mathrm{mg} / \mathrm{dL}(141.44$ $\mathrm{mol} / \mathrm{L})$ that improved to $0.8 \mathrm{mg} / \mathrm{dL}(70.72 \mathrm{~mol} / \mathrm{L})$ on hospital day 3. Electrolytes on admission were all within normal ranges:

\section{Table 1: Patient's Arterial Blood Gases}

\begin{tabular}{lcccc}
\hline & $\begin{array}{c}\# \mathbf{1} \\
\text { (9 hours) }^{*}\end{array}$ & $\begin{array}{c}\text { \#2 } \\
\text { (12 hours) }\end{array}$ & $\begin{array}{c}\text { (12.5 } \\
\text { (120urs) }\end{array}$ & $\begin{array}{c}\text { \#4 } \\
\text { (28 hours) }\end{array}$ \\
\hline $\mathrm{pH}$ & 7.45 & 7.48 & 7.52 & 7.5 \\
\hline $\mathrm{pCO} 2$ & 43 & 46 & 40 & 43 \\
\hline $\mathrm{HCO} 3$ & 29.9 & 34.3 & 32.7 & 33.5 \\
\hline $\mathrm{BE}$ & 5.3 & 9.5 & 9.1 & 9.3 \\
\hline
\end{tabular}

*Number of hours after arrival at toxicology center

Site: right radial stick (all values listed)

sodium (142 mmol/L), potassium (4.2 mmol/L), chloride (109 $\mathrm{mmol} / \mathrm{L})$, and CO2 (27.1 mmol/L). Initial CK was $368 \mathrm{U} / \mathrm{L}$, improving to $172 \mathrm{U} / \mathrm{L}$ on hospital day 2 . Complete blood count showed a leukocytosis of $21,05010^{9} / \mathrm{L}$, decreasing to $9,39010^{9} / \mathrm{L}$ on hospital day 2 . There was a mild elevation of his troponin to $0.18 \mathrm{~g} / \mathrm{L}$ (normal range $0.0-0.03 \mathrm{~g} / \mathrm{L}$ ). This normalized over the first day in the ICU and it likely related to attempted cardioversions. His drug screen was negative for all illicit drugs, but was positive for his daily medications.

Once his normal mental state was achieved, the patient admitted to researching yew berry toxicology on the Internet. However, he told the consulting psychiatrist that he had just happened upon the yew berry plant. The patient was insistent that he had ingested 168 yew berry seeds. Most seeds were chewed, while others were ingested intact. Any error in the count would be less than 15 seeds. The patient denied any co-ingestion. Of note, the patient's parents brought seeds in for identification purposes to aid

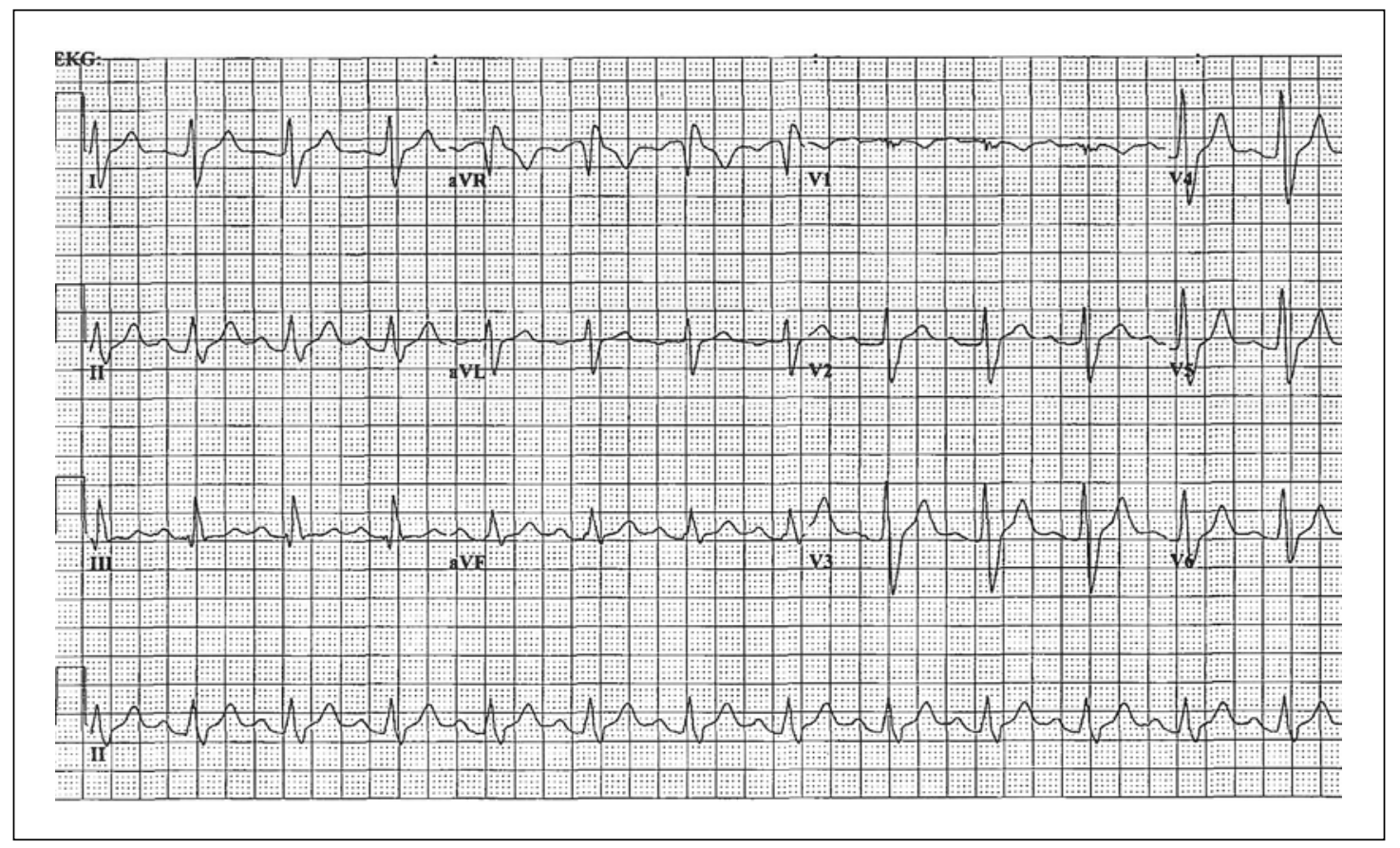

Figure 4: EKG after initiation of bicarbonate infusion 


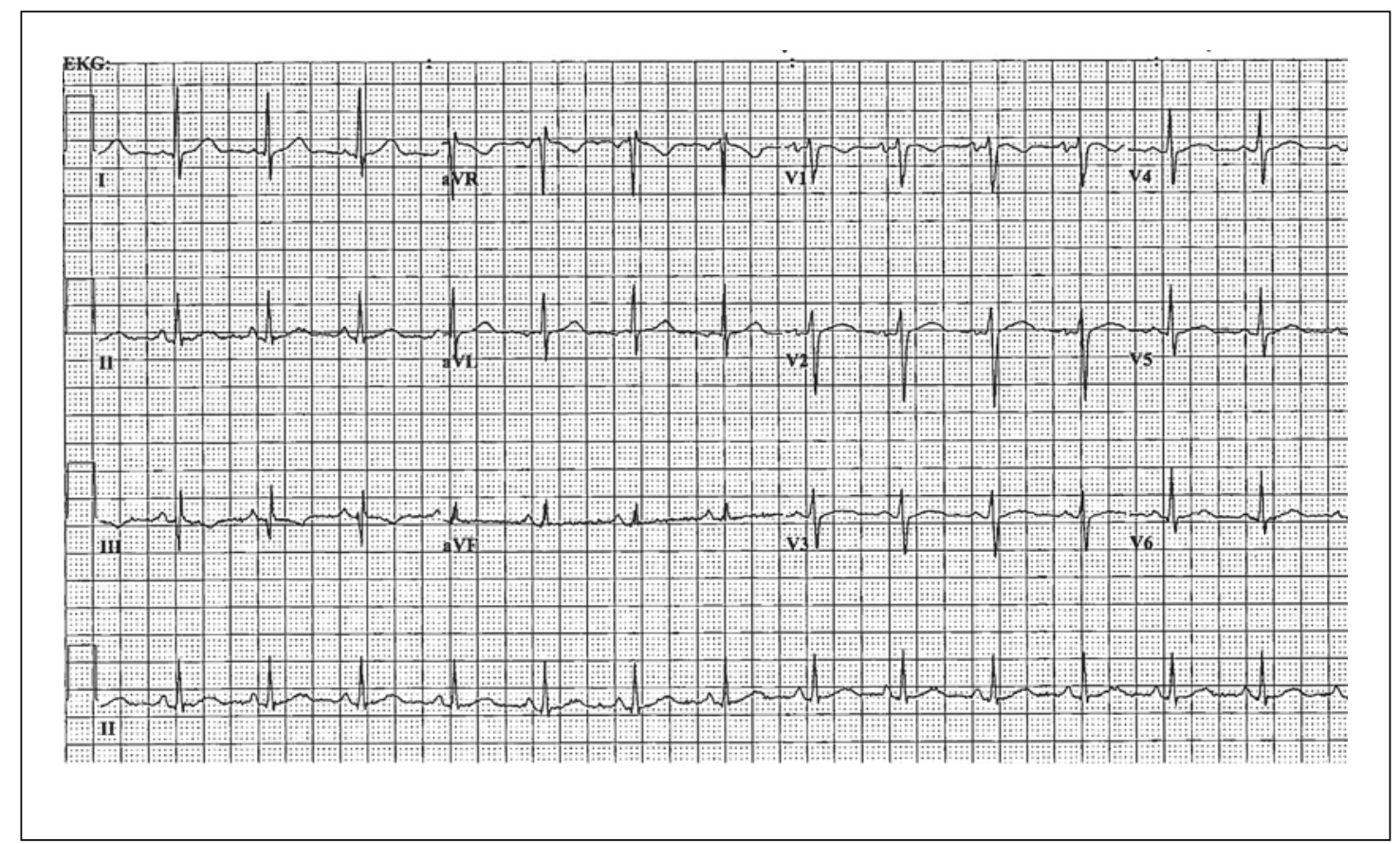

Figure 5: EKG upon discharge from the toxicology center

in treatment; they are botanists and confirmed the identification of Taxus cuspidata.

\section{DISCUSSION}

Taxus cuspidate (Japanese yew or spreading yew) is a member of the genus Taxus. Taxus toxicity from ingestion is rare because most individuals do not chew the seeds and as such do not release the toxin, yet ingestion can result in death. The Japanese yew is dedicated to the "Gods of death" and is more commonly referred to as "the tree of death." Taxine toxins are found in the seeds and leaves, but not the berries surrounding the seeds [2]. The toxins have predominantly cardiotoxic and gastrointestinal effects. Rapidly metabolized by the liver, excreted as benzoic acid, fatalities in animals have occurred within minutes of ingestion [3]. By far, the vast majority of deaths occur in livestock, with $1 \mathrm{~g}$ of yew leaves containing $5 \mathrm{mg}$ of taxine [4].

Taxine toxins cause cardiac dysrhythmias secondary to interference with the sodium-potassium transport system. Currently, there is no known effective treatment or antidote, and classic antiarrhythmic therapy has proven to be ineffective [5-8]. According to studies by Alloatti et al., taxine B prolongs AV conduction, and prolongs QRS duration time, ultimately resulting in 2nd- and 3rd-degree heart block and diastolic cardiac arrest [9]. Further electrophysiologic studies by Tekol and Kameyama in 1987 found that the taxine toxins inhibit both calcium and sodium channels in a dose-dependent manner $[4,6]$. The sodium channel blockade of the taxine toxins is similar to that seen with class 1 antiarrhythmics such as quinidine, procainamide, and disopyramide, prolonging the QT interval and widening the QRS. The mecha- nism of calcium channel blockade exhibited by taxine toxins is much like that seen in class 4 antiarrhythmics such as amlodipine, diltiazem, verapamil, and nicardipine-affecting phase 2 of the action potential, causing heart block, slowing AV conduction, and prolonging the PR interval. Additionally, the taxine toxins are structurally similar to digitalis. Digitalis competes with potassium for a binding site on the cardiac membrane, poisoning the sodium-potassium pump and ultimately causing an increase in intracellular calcium. Because of the structural similarity with digitalis, digoxin-specific antibody fragments have been studied as possible treatment for taxine toxicity. Previous study has shown this treatment approach to be ineffective [10].

Ruha et al. utilized hypertonic saline to treat Taxus cardiac toxicity in swine, but found it ineffective in reversing widened QRS interval in this animal model [7]. Miller et al. published a case report of yew berry ingestion and other co-ingestants (paroxetine, brodificoum, and shellae) successfully treated with a sodium bicarbonate drip in combination with pressors (epinephrine, dopamine and norepinephrine) [11]. Mechanistically, sodium bicarbonate is used to reverse acidosis, raise $\mathrm{pH}$, and increase sodium conductance through myocardial sodium channels.

In this case, successful treatment of the patient included amiodarone followed by sodium bicarbonate bolus and infusion, demonstrating direct narrowing of the QRS. It is difficult to determine if correction of the cardiac dysrhythmias was solely due to the sodium bicarbonate, or the synergism of sodium bicarbonate and amiodarone, or possible spontaneous improvement due to taxine clearance. Administration of sodium bicarbonate improved both his clinical status and telemetry recording within 
minutes. This improvement is documented in the serial EKGs. The half-life of sodium bicarbonate IV is extremely short, and successful treatment may rely on a continuous infusion. Therefore, based on the clinical improvement of this patient, both with EKG recordings and vital signs, this report would suggest that isolated taxine ingestion from yew berry plants could be treated with sodium bicarbonate. Obviously, there are concerns about drawing conclusions from a single case; however, the single toxin ingested in this case potentially provides a more accurate picture than other case reports.

The authors have no potential financial conflicts of interest to report.

\section{REFERENCES}

1. Nora M, Elsner G, Purdy C, et al. Wide QRS rhythm due to taxine toxicity. J Cardiovasc Electrophysiol 1993;3:59-61.

2. Wilson CR, Sauer JM, Hooser SB. Taxines: a review of the mechanism and toxicity of yew (Taxus spp.) alkaloids. Toxicon 2001;39:175-185.

3. Lowe JE, Hintz HF, Schryver HF, et al. Taxus cuspidata (Japanese yew) poisoning in horses. Cornell Vet 1970;60(1):36-39.

4. Tekol Y, Gõgüsten B. Comparative determination of the cardiosensivity of taxine and verapamil in the isolated aorta, atrium, and jejunum preparations of rabbits. Arzneim-Forschung 1999;49(8):673-678.

5. Musshoff F, Jacob B, Fowinkel C, et al. Suicidal yew leaf ingestion-phloroglucindimethylether (3,5-dimethoxyphenol) as a marker for poisoning from Taxus baccata. Int J Legal Med 1993;106(1):45-50.

6. Tekol Y, Kamayama M. Elektrophysiologische Untersuchungen über den Wirkungsmechanisms des Eibentoxin Taxin auf das Herz. Arzneim.-Forschun. 1987;37(4):428-431.

7. Ruha AM, Tanen DA, Graeme KA, et al. Hypertonic sodium bicarbonate for Taxus media-induced cardiac toxicity in swine. Acad Emerg Med 2002;9(3):179-185.

8. Gustafson DL, Long ME, Zirrolli JA, et al. Analysis of doctaxel pharmacokinetics in humans with the inclusion of later sampling time-points afforded by the use of a sensitive tandem LCMS assay. Cancer Chemother Pharmacol 2003;52(2):159-166.

9. Alloatti G, Penna C, Levi RC, et al. Effects of yew alkaloids and related compounds on guinea-pig isolated perfused heart and papillary muscle. Life Sci 1996;58(10):845-854.

10. Cummins RO, Haulman J, Quan L, et al. Near fatal yew berry intoxication treated with external cardiac pacing and digoxin-specific FAB fragments. Ann Emerg Med 1990;19:38-43.

11. Miller MB, Eng J, Curry SC. Sodium bicarbonate for taxusinduced dysrhythmia. Ingham Regional Medical Center, Dewitt, MI. NACCT Abstracts, 2000. 